Article

\title{
Characterization of the Volatile Compounds in Camellia oleifera Seed Oil from Different Geographic Origins
}

\author{
Jing Wang ${ }^{1}$, Xuxiao Tang ${ }^{1}$, Qiulu Chu ${ }^{2}$, Mengyu Zhang ${ }^{1}$, Yingzhong Zhang ${ }^{1, *}$ and Baohua Xu ${ }^{1}$ \\ 1 Guangdong Provincial Key Laboratory of Silviculture, Protection and Utilization, Guangdong Academy \\ of Forestry, Guangzhou 510520, China; wangjing@sinogaf.cn (J.W.); tangxuxiao666@163.com (X.T.); \\ rainbow84397520@163.com (M.Z.); baohuaxu@aliyun.com (B.X.) \\ 2 Jiangsu Co-Innovation Center of Efficient Processing and Utilization of Forest Resources, College of Light \\ Industry and Food Engineering, Nanjing Forestry University, Nanjing 210037, China; chuqiulu@njfu.edu.cn \\ * Correspondence: zyzh3762@sinogaf.cn; Tel.: +86-020-8707-1272
}

check for updates

Citation: Wang, J.; Tang, X.; Chu, Q.; Zhang, M.; Zhang, Y.; Xu, B. Characterization of the Volatile Compounds in Camellia oleifera Seed Oil from Different Geographic Origins. Molecules 2022, 27, 308. https: / /doi.org/10.3390/ molecules27010308

Academic Editor: Chiara Emilia Cordero

Received: 28 November 2021

Accepted: 1 January 2022

Published: 4 January 2022

Publisher's Note: MDPI stays neutral with regard to jurisdictional claims in published maps and institutional affiliations.

Copyright: (C) 2022 by the authors. Licensee MDPI, Basel, Switzerland. This article is an open access article distributed under the terms and conditions of the Creative Commons Attribution (CC BY) license (https:// creativecommons.org/licenses/by/ $4.0 /)$.

\begin{abstract}
Volatile flavor of edible oils is an important quality index and factor affecting consumer choice. The purpose of this investigation was to characterize virgin Camellia oleifera seed oil (VCO) samples from different locations in southern China in terms of their volatile compounds to show the classification of VCO with respect to geography. Different samples from 20 producing VCO regions were collected in 2020 growing season, at almost the same maturity stage, and processed under the same conditions. Headspace solid-phase microextraction (HS-SPME) with a gas chromatographymass spectrometer system (GC-MS) was used to analyze volatile compounds. A total of 348 volatiles were characterized, including aldehydes, ketones, alcohols, acids, esters, alkenes, alkanes, furans, phenols, and benzene; the relative contents ranged from $7.80-58.68 \%, 1.73-12.52 \%, 2.91-37.07 \%$, $2.73-46.50 \%, 0.99-12.01 \%, 0.40-14.95 \%, 0.00-27.23 \%, 0.00-3.75 \%, 0.00-7.34 \%$, and $0.00-1.55 \%$, respectively. The VCO geographical origins with the largest number of volatile compounds was Xixiangtang of Guangxi (L17), and the least was Beireng of Hainan (L19). A total of 23 common and 98 unique volatile compounds were detected that reflected the basic and characteristic flavor of VCO, respectively. After PCA, heatmap and PLS-DA analysis, Longchuan of Guangdong (L8), Qingshanhu of Jiangxi (L16), and Panlong of Yunnan (L20) were in one group where the annual average temperatures are relatively low, where annual rainfalls are also low. Guangning of Guangdong (L6), Yunan of Guangdong (L7), Xingning of Guangdong (L9), Tianhe of Guangdong (L10), Xuwen of Guangdong (L11), and Xiuying of Hainan (L18) were in another group where the annual average temperatures are relatively high, and the altitudes are low. Hence, volatile compound distributions confirmed the differences among the VCO samples from these geographical areas, and the provenance difference evaluation can be carried out by flavor.
\end{abstract}

Keywords: Camellia oleifera seed oil; geographical classification; HS-SPME/GC-MS; volatile compounds

\section{Introduction}

Camellia oleifera, a kind of theaceous evergreen shrub tree or middle arbor, has been cultivated for more than 2300 years in central and southern regions of China that also distributes in Japan and other Southeast Asia areas [1,2]. C. oleifera seed oil contains squalene [3], sterol [3-5], tocopherol [5], polyphenols [6], and a high content ( $\geq 90 \%)$ of unsaturated fatty acids (mainly oleic acids and linoleic acid) [7,8], and plays important roles in antioxidant $[9,10]$, anti-inflammatory [11], hepatoprotective, and gastroprotective functions [12]. Virgin C. oleifera seed oil (VCO) belongs to a natural product and is obtained by mechanical or physical methods without any further refining process. The VCO contains many components that are favorable in terms of health. One of the most important reasons is the volatile compounds [13] that are principally generated by fatty acid oxidation and have great concern because of their impact on the quality of foods and the sensory attributes. 
The specific flavor of VCO is also one of the many factors considered separately from the other edible vegetal oils.

The volatile compounds of edible oil comprise of several short-chain hydrocarbons or a minimal number of polar functional groups with good nonpolar features, such as aldehydes, alcohols, ketones, acids, furan, phenols, and esters [14]. They are commonly responsible for the characteristic flavor of edible vegetal oil, which plays a significant role in the food industry because it has prime influence on consumer choice [15-17]. However, in hundreds of different volatile compounds, only a small fraction actually contributes to the overall flavor [18]. Hence, the volatile profiles can be used to estimate the quality of VCO and identify the variety of Camellia.

In recent years, gas chromatography-mass spectrometry (GC-MS), electronic nose (E-nose), gas chromatography-olfactometry (GC-O), and so on have been widely developed in the food and pharmaceutical industry $[19,20]$. Among them, GC-MS analysis has the excellent ability of simultaneously separating, identifying, even quantifying the multiple volatile components [21,22].

The flavor composition and nutritional evaluation of edible plants in different growing locations has been an important research field in the past few years, such as focusing on volatile compounds, fatty acids, amino acids, polyphenols, and antioxidant activities in Capsicum annuum [23], Viburnum opulus L. [24], olive [18,25-27], Taxus Baccata L. [28], Paeonia ostii [29], and Camellia sinensis [30]. It has long been known that the volatile compounds of edible oil are related to genetic (cultivars) [31-35], environmental (geography $[18,25,27,31]$, climatic conditions [27,31] and storage conditions [31]), cultivating (agronomic techniques [36] and the degree of fruit ripening [31,36]), and processing (harvesting methods [31] and processing technology [37]) factors. Therefore, geographic origin of C. oleifera is greatly responsible for the sensorial characteristics of VCO. Moreover, the volatile compounds of oil obtained from different production areas, under identical growth conditions, harvested at roughly equal ripeness degree, and processed in the same manner, can be characterized by different compositions and their respective concentrations. There has been increasing interest in the geographical identification of virgin plant oil, as a reliable criterion for its authentication and quality [25].

However, until now, the research on the differences of VCO flavor characteristics mainly focuses on processing treatment $[1,38]$. There is little information on the identification of the main odorants in China VCO from different geographic origins. Therefore, the aim of this study was to investigate the characterization of the volatile compounds extracted from VCO produced in different geographical locations of southern China by GC-MS with multivariate statistical methods, and to establish the specific volatile substances or their categories that probably affect the VCO flavor from different geographical regions.

\section{Results and Discussion}

\subsection{Comprehensive Analysis of Volatile Compounds}

Geographical factors have a strong effect on the formation of chemically volatile substances of Camellia oil [34], especially for virgin oil [35]. In order to evaluate the characterization of the flavor of virgin Camellia oleifera seed oil (VCO) from different planting locations in southern China, HS-SPME/GC-MS was performed to analyze the volatile compounds in this study. The ion total chromatogram curves of $20 \mathrm{VCO}$ samples are demonstrated in Figure 1. The number and retention times of the ion chromatographic peaks represent the differences of volatile compounds of VCO. In L1 to L20 samples, L4 showed the largest number of peaks, which indicates that it might contain more quantity of volatile compounds. Moreover, L16 displayed the longest retention time span and it had an obvious abundance at $47.50 \mathrm{~min}$ that was hexatriacontane by system software analysis. It could be intuitively reflected from Figure 1 that there are differences in the types and quantities of volatile components of $20 \mathrm{VCO}$ from different geographic regions. Hence, the number and content of volatile components of all VCO samples will be further studied. 


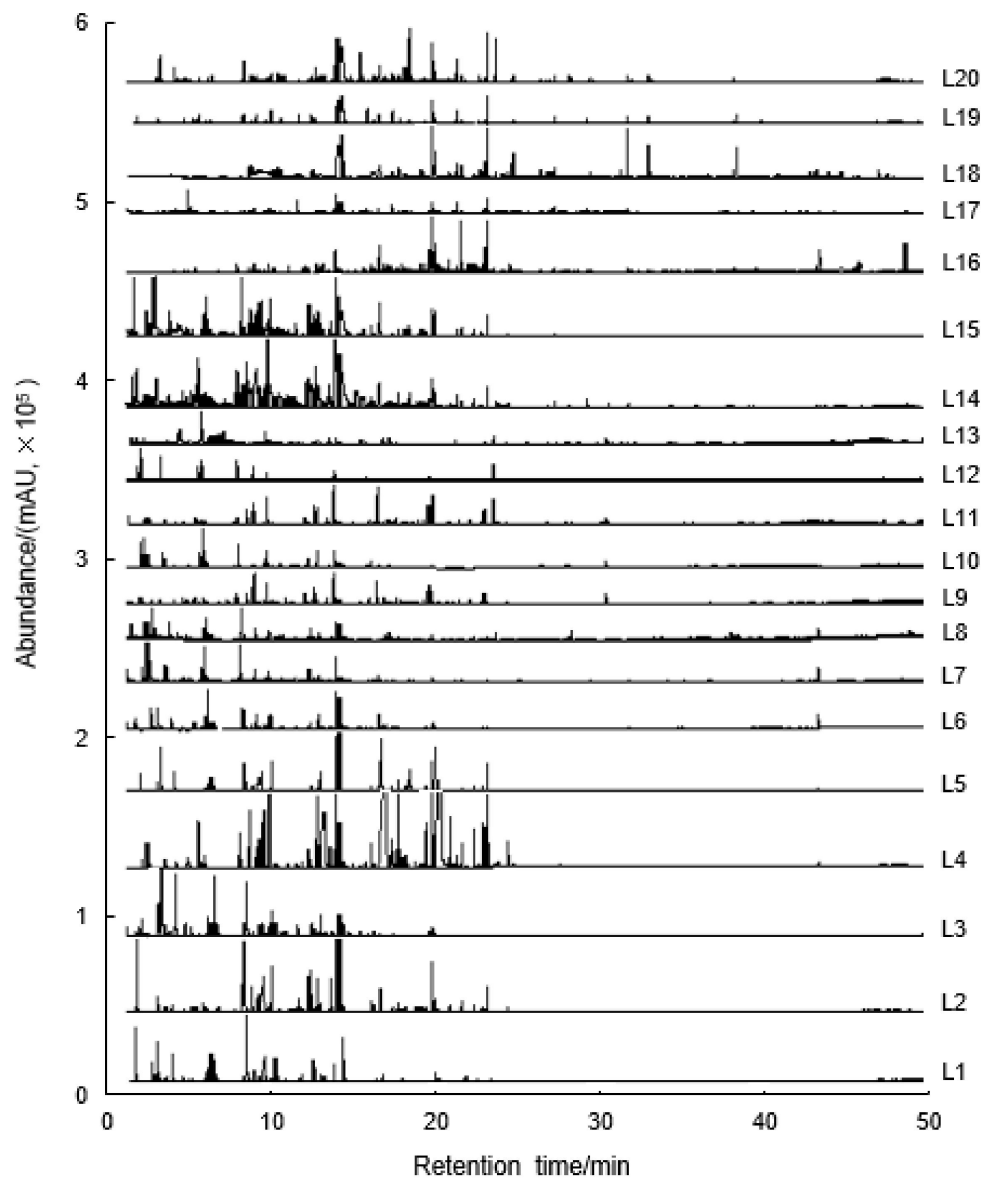

Figure 1. Total ion chromatograms of volatile compounds of virgin Camellia oleifera seed oil (VCO) from 20 geographic regions.

\subsection{Composition Analysis of Volatile Components}

2.2.1. Analysis of the Contents and Quantity of Volatile Compounds

Not only can threshold values of specific volatile compounds affect the flavor of edible oil to a certain extent [17], the type and content of flavor substances also play an important function in the odorant. The analysis result by HS-SPME/GC-MS shows that a total of 348 volatile compounds were identified in $20 \mathrm{VCO}$ samples. According to different properties, the volatiles of VCO samples are divided into 10 chemical categories (Figure 2), namely ketones, alcohols, alkanes, esters, aldehydes, alkenes, acids, phenols, furans, and benzenes, which play significant roles in the discrimination of the VCO.

Among them, ketones (69) were the most abundant volatile compounds in this study (Figure 2A); these are formed by auto-oxidation of fatty acids, $\beta$-oxidation, and decarboxylation [39], and usually give the sweet and fruity flavor. Alcohols (57) and aldehydes (48), also as main compounds in all VCO samples, being formed by oxidative degradation of fatty acids or Strecker degradation of amino acids [38], contributed the overall odor of Camellia oil with large quantity; these findings are consistent with a previous report [34]. A total of 51 esters were identified that can be produced by esterification of alcohols with free small molecular fatty acids, or by enzymatic degradation of amino acids during the growth of Camellia oleifera. Most esters are described as having fruit and flower aromas. In addition to the above four kinds of substances, alkanes (51) and alkenes (48) were also main types of volatile components and play a key role in the overall flavor of VCO. The remaining number of acids, phenols, furans, and benzenes were $24,9,8$, and 4, respectively. These multivariate compounds and their interactions constitute the complicated flavor of VCO. 
A)

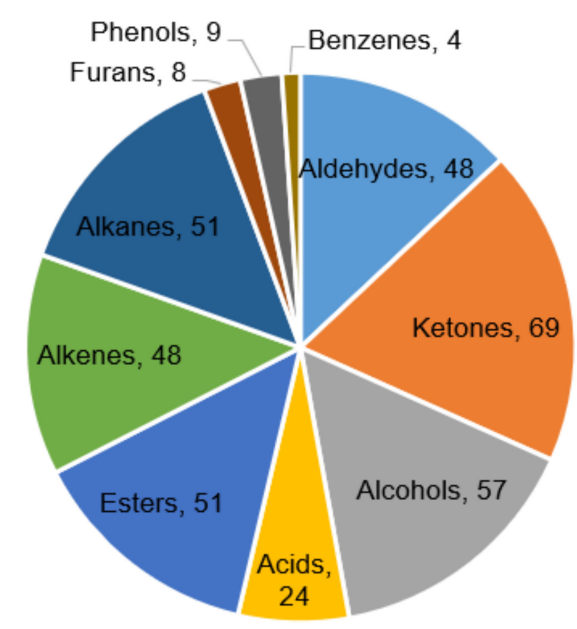

B)

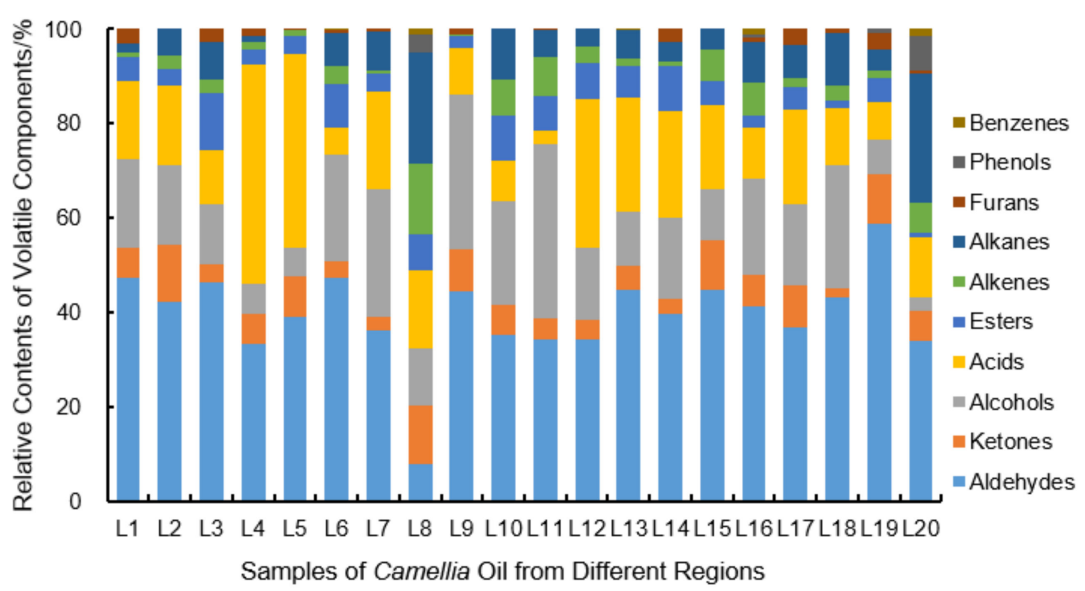

C)

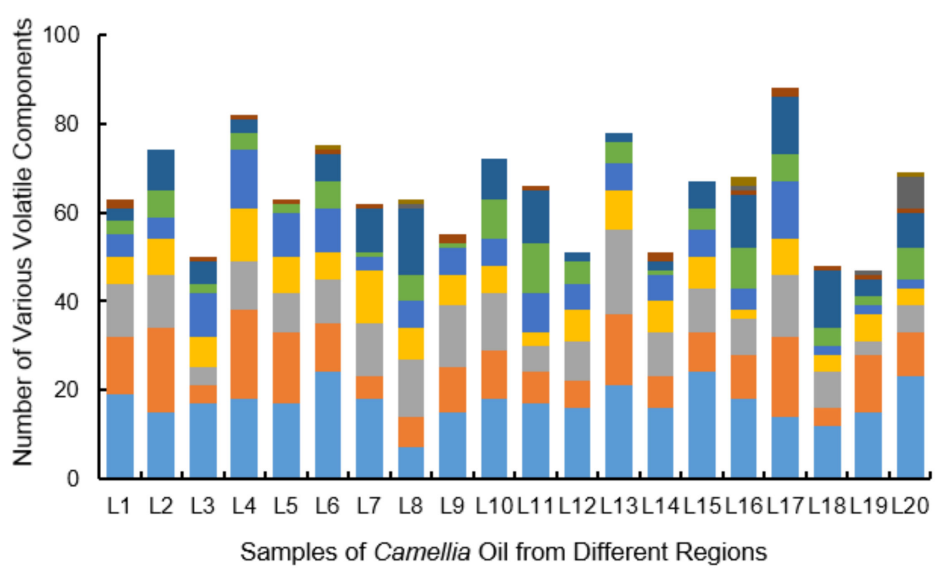

Figure 2. The composition and content of volatile compounds of VCO from 20 geographic regions. (A) The number of ketones, alcohols, alkanes, esters, aldehydes, alkenes, acids, phenols, furans, and benzenes in all Camellia oleifera seed oils. (B) The relative contents of 10 chemical categories of volatiles in VCO samples from 20 regions. (C) The number of various volatile components in VCO samples from 20 regions.

VCO from different planting areas usually presents a unique flavor, which is closely related to the relative proportion and number of flavor substances. Hence, the relative 
percentage contents of $\mathrm{VCO}$ volatile components quantitatively calculated by peak area normalization method (Figure 2B) and the quantities (Figure 2C) are discussed in this study. As shown in Figure 2B, the highest proportions of aldehydes and furan were detected in L19 (58.68\% and 3.75\%, respectively), ketones and alkenes in L8 (12.52\% and $14.95 \%$, respectively), alcohols in L11 (37.07\%), acids in L4 (46.50\%), esters in L3 (12.01\%), and alkanes, phenols, and benzenes in L20 (27.23\%, $7.34 \%$, and $1.55 \%$, respectively). Some predominant aldehydes. such as furfural and (Z)-13-octadecenal, had particularly high proportion, which reached $31.38 \%$ in L18 and $24.29 \%$ in L6, respectively (data not shown in figure). Octanoic acid also accounted for high levels in the L4 sample with a relative content of $22.60 \%$, which usually has a strong fat flavor at high concentrations [34]. It has been reported that geographical origin is an important factor affecting the phenolic formation [26]. However, in this study, phenolic compounds could be found only in L8, L16, L19, and L20, and not in the other 16 samples.

From Figure 2C, the number of volatile compounds in L17 oil (88) was 1.07-1.87 times higher compared to other locations samples. It is worth mentioning that the aldehyde with the highest content was in the L19 oil, but the quantity (15) in this oil was not a maximum. The largest number of aldehydes was 24, both in L6 and L15. The highest content of ketones was in L8; nevertheless, the largest number was in L2. L11 had the highest content of alcohols, the number of which in L11 sample was six. Although the number of alcohols in L20 was also six, its content was the lowest in all samples, at only $2.91 \%$. There is no correlation between the contents and quantity of various compounds in each sample, but they both show that the main types of volatile substances in VCO are aldehydes, ketones, alcohol, acids, esters, alkenes, and alkanes. In the study of other food flavors with regard to geographical origins, aldehydes and alcohols are considered the largest volatile profiles in olive oil with the 2-hexenal and 1-hexanol more than 50\% [18,27] and 12\% [25], respectively. Alkanes are the main volatile compounds in bell pepper spices [23], and acids and ketones are the main volatile compounds in Viburnum opulus L. fruits [24]. Compared to other oils or plants, VCO presents its own special flavor.

The formation of different odorants does not necessarily result from a high number of volatile compounds [40]. Nevertheless, our study revealed that the relative content and quantity of volatile compounds of VCO in different locations have their own characteristics. This specificity together could constitute the uniqueness of the flavor of each VCO sample. Therefore, this study will further classify VCO by multivariate statistical analysis to find the similarity and regularity of VCO from 20 geographic regions.

\subsubsection{Analysis of the Common and Unique Volatile Components}

After analysis of all the VCO samples from different geographic regions, a total of 23 common volatile components were detected, including 12 of aldehydes, 1 of ketones, 3 of alcohols, 4 of acids, 1 of esters, 1 of alkenes, and 1 of phenols (Table 1). These compounds construct the basic flavor of VCO. Among them, decanal with sweet flavor, 2,4-decadienal with deep-fried flavor, (E)-2-decenal with fatty flavor, and 2-undecenal with fresh aldehyde flavor [41], were found in all of VCO samples and their retention indexes were approximate (1204-1311), suggesting that these flavors are highly common in VCO. It is particularly noteworthy that they are all aldehydes and the specific reason for this occurrence pattern remains to be determined. Hexanal was also a common compound of aldehydes and found in 19 VCO samples, which is consistent with the previous research [38,42]. It is produced by linoleic acid oxidation and shows high content in tea seed oil, grape seed oil, soybean oil, and corn oil [34]. The analysis of common volatile compounds confirms again that aldehydes, which universally exist in olive oil [18], are also major and important volatile compounds in VCO.

Except for aldehydes, acids were also main common volatile substances of VCO, including hexanoic acid, 4-hydroxy-butanoic acid, nonanoic acid, and octanoic acid, which is consistent with previous reports [33]. In four of the common acids, hexanoic and octanoic acid show a VCO sensory description of sweety and fatty that matches the flavor of decanal 
and (E)-2-decenal. Nonanoic acid is characterized by cheese and sweet that also could be identified as the volatile compounds of most fruits [38]. It is noteworthy that L8 sample had not common acids that probably implies the specific of L8.

Table 1. Information of common volatile compounds of VCO from 20 geographic regions.

\begin{tabular}{|c|c|c|c|c|c|c|}
\hline NO. & Volatile Compound & CAS & Formula & $\begin{array}{l}\text { Retention } \\
\text { Index }\end{array}$ & Sensory Descriptions ${ }^{a}$ & $\begin{array}{l}\text { Unrecognized } \\
\text { Samples }\end{array}$ \\
\hline & Aldehydes & & & & & \\
\hline 1 & Decanal & $112-89-0$ & $\mathrm{C}_{10} \mathrm{H}_{20} \mathrm{O}$ & 1204 & Sweet, waxy & - \\
\hline 2 & 2,4-Decadienal & $2363-88-4$ & $\mathrm{C}_{10} \mathrm{H}_{16} \mathrm{O}$ & 1220 & Deep-fried & - \\
\hline 3 & (E)-2-Decenal & $3913-81-3$ & $\mathrm{C}_{10} \mathrm{H}_{18} \mathrm{O}$ & 1212 & Fatty, green & - \\
\hline 4 & 2-Undecenal & $2463-77-6$ & $\mathrm{C}_{11} \mathrm{H}_{20} \mathrm{O}$ & 1311 & Strong fresh aldehyde & - \\
\hline 5 & Hexanal & $66-25-1$ & $\mathrm{C}_{6} \mathrm{H}_{12} \mathrm{O}$ & 806 & Cut grassy, apple & L8 \\
\hline 6 & (E)-2-Nonenal & $18829-56-6$ & $\mathrm{C}_{9} \mathrm{H}_{16} \mathrm{O}$ & 1112 & Green, fatty & L8 \\
\hline 7 & Octanal & 124-13-0 & $\mathrm{C}_{8} \mathrm{H}_{16} \mathrm{O}$ & 1005 & Vanilla, orange & L19 \\
\hline 8 & Heptanal & $111-71-7$ & $\mathrm{C}_{7} \mathrm{H}_{14} \mathrm{O}$ & 905 & Green plant, oily & L8, L18 \\
\hline 9 & Nonanal & $124-19-6$ & $\mathrm{C}_{9} \mathrm{H}_{18} \mathrm{O}$ & 1104 & Grassy, Almond & L11, L14 \\
\hline 10 & (Z)-2-Heptenal & $57266-86-1$ & $\mathrm{C}_{7} \mathrm{H}_{12} \mathrm{O}$ & 913 & Oxidised, pungent & L14, L15, L19 \\
\hline 11 & Furfural & 98-01-1 & $\mathrm{C}_{5} \mathrm{H}_{4} \mathrm{O}_{2}$ & 831 & Almond & L8, L15, L16, L20 \\
\hline 12 & $\begin{array}{l}(\text { E)-2-Octenal } \\
\text { Ketones }\end{array}$ & $2548-87-0$ & $\mathrm{C}_{8} \mathrm{H}_{14} \mathrm{O}$ & 1013 & Green, floral & L3, L8, L16, L19 \\
\hline 13 & $\begin{array}{l}\gamma \text {-Octanoic lactone } \\
\text { Alcohols }\end{array}$ & $104-50-7$ & $\mathrm{C}_{8} \mathrm{H}_{14} \mathrm{O}_{2}$ & 1184 & Peach, coconut, oatmeal bread & L3, L6, L18 \\
\hline 14 & 1-Heptanol & $111-70-6$ & $\mathrm{C}_{7} \mathrm{H}_{16} \mathrm{O}$ & 960 & Fresh, woody & L15, L16, L19 \\
\hline 15 & 2-Furan methanol & $98-00-0$ & $\mathrm{C}_{5} \mathrm{H}_{6} \mathrm{O}_{2}$ & 885 & Bitterness & L8, L15, L16, L20 \\
\hline 16 & $\begin{array}{l}\text { Benzyl alcohol } \\
\text { Acids }\end{array}$ & $100-51-6$ & $\mathrm{C}_{7} \mathrm{H}_{8} \mathrm{O}$ & 1036 & Aromatic & L5, L8, L12, L18, L19 \\
\hline 17 & Octanoic acid & $124-07-2$ & $\mathrm{C}_{8} \mathrm{H}_{16} \mathrm{O}_{2}$ & 1173 & Oily, fatty & L8 \\
\hline 18 & Hexanoic acid & $142-62-1$ & $\mathrm{C}_{6} \mathrm{H}_{12} \mathrm{O}_{2}$ & 974 & Sweet, pungent & L8, L20 \\
\hline 19 & Nonanoic acid & $112-05-0$ & $\mathrm{C}_{9} \mathrm{H}_{18} \mathrm{O}_{2}$ & 1272 & Cheese, sweet & L8, L9, L18 \\
\hline 20 & $\begin{array}{l}\text { 4-Hydroxybutanoic acid } \\
\text { Ester }\end{array}$ & $591-81-1$ & $\mathrm{C}_{4} \mathrm{H}_{8} \mathrm{O}_{3}$ & 1018 & Buttery, rancid & L8, L15, L16, L20 \\
\hline 21 & $\begin{array}{l}\text { Methyl cinnamate } \\
\text { Alkenes }\end{array}$ & $103-26-4$ & $\mathrm{C}_{10} \mathrm{H}_{10} \mathrm{O}_{2}$ & 1267 & Cherry, balsamic flavor & L4, L9, L14, L20 \\
\hline 22 & $\begin{array}{l}\text { 8-Methyl-1-undecene } \\
\text { Phenols }\end{array}$ & $74630-40-3$ & $\mathrm{C}_{12} \mathrm{H}_{24}$ & 1140 & NF & L8, L10, L18, L20 \\
\hline 23 & Maltol & $118-71-8$ & $\mathrm{C}_{6} \mathrm{H}_{6} \mathrm{O}_{3}$ & 1063 & Caramel & L2, L4, L8, L17, L20 \\
\hline
\end{tabular}

Note: ${ }^{\text {, }}$ Sensory descriptions were obtained from Fenaroli's Handbook of Flavor Ingredients [41]. NF, not found.

In addition, the benzyl alcohol, 2-furan methanol and 1-heptanol as common alcohols are contributed to the overall flavor of $\mathrm{VCO}$ with aromatic, bitterness, and woody. The other common volatile compounds of ketones, esters, alkenes, and phenols were 5-butyldihydro2(3H)-furanone, 3-phenyl-2-propenoic acid methyl ester, 8-methyl-1-undecene, and maltol, respectively. Maltol (3-hydroxy-2methyl-4-pyrone), which is usually found in roasted cocoa powder as the volatile flavor compound of caramel [43], was also not recognized in the L8 sample.

If the common volatile compounds show the basic flavor of $\mathrm{VCO}$, the unique volatile components in each VCO sample may be one of the factors influencing the formation of characteristic flavor. Table 2 lists a total of 98 unique volatile compounds detected in VCO from different geographic regions. The sensory descriptions of the unique volatile substances are not shown in Table 2, because most of detected unique volatile compounds have only a few studies. The number of unique aldehydes, ketones, alcohols, acids, esters, alkenes, alkanes, furans, and phenols were 5, 23, 11, 9, 13, 16, 13, 4, and 4, respectively. And the number of unique volatile components tested in L1-L20 samples were $\mathbf{2 , 4}, \mathbf{4}, \mathbf{1 4}, \mathbf{3}, \mathbf{6 ,} \mathbf{2}$, $7,4,7,8,0,7,2,7,3,3,0,7$, and 8 , respectively. 
Table 2. Information of unique volatile compounds of VCO from 20 geographic regions.

\begin{tabular}{|c|c|c|c|c|c|c|}
\hline NO. & Volatile Compound & CAS & Formula & $\begin{array}{l}\text { Retention } \\
\text { Index }\end{array}$ & $\begin{array}{c}\text { Similarity } \\
(\%)\end{array}$ & $\begin{array}{c}\text { Recognized } \\
\text { Sample }\end{array}$ \\
\hline & Aldehydes & & & & & \\
\hline 1 & 2,3-Dihydro-4-carboxaldehyde & $37414-43-0$ & $\mathrm{C}_{10} \mathrm{H}_{10} \mathrm{O}$ & 1348 & 81 & L6 \\
\hline 2 & 3-Hydroxy-4-methoxy-benzaldehyde & $621-59-0$ & $\mathrm{C}_{8} \mathrm{H}_{8} \mathrm{O}_{3}$ & 1392 & 90 & L20 \\
\hline 3 & (Z)-13-Octadecenal & $58594-45-9$ & $\mathrm{C}_{18} \mathrm{H}_{34} \mathrm{O}$ & 2007 & 86 & L6 \\
\hline 4 & 13-Tetradecenal & $85896-31-7$ & $\mathrm{C}_{14} \mathrm{H}_{26} \mathrm{O}$ & 1591 & 80 & L10 \\
\hline 5 & $\begin{array}{l}\text { (Z)-4-Undecenal } \\
\text { Ketones }\end{array}$ & $68820-32-6$ & $\mathrm{C}_{11} \mathrm{H}_{20} \mathrm{O}$ & 1311 & 88 & L15 \\
\hline 6 & $\gamma$-Butyrolactone & $96-48-0$ & $\mathrm{C}_{4} \mathrm{H}_{6} \mathrm{O}_{2}$ & 825 & 85 & L19 \\
\hline 7 & Cyclopentadecanone & $502-72-7$ & $\mathrm{C}_{15} \mathrm{H}_{28} \mathrm{O}$ & 1970 & 85 & L6 \\
\hline 8 & 1,4-Cyclooctanedione & $55794-45-1$ & $\mathrm{C}_{8} \mathrm{H}_{12} \mathrm{O}_{2}$ & 1302 & 84 & L4 \\
\hline 9 & $\begin{array}{l}\text { 2,3-Dihydro-3,5-dihydroxy-6-methyl- } \\
\text { 4(4H)-pyranone }\end{array}$ & 29446-10-4 & $\mathrm{C}_{6} \mathrm{H}_{8} \mathrm{O}_{4}$ & 1269 & 92 & L19 \\
\hline 10 & Dihydro-5-methyl-3(2H)-furanone & $34003-72-0$ & $\mathrm{C}_{5} \mathrm{H}_{8} \mathrm{O}_{2}$ & 821 & 83 & L11 \\
\hline 11 & 3-Nonanone & $925-78-0$ & $\mathrm{C}_{9} \mathrm{H}_{18} \mathrm{O}$ & 1053 & 93 & L13 \\
\hline 12 & 4-Dodecanone & $6137-26-4$ & $\mathrm{C}_{12} \mathrm{H}_{24} \mathrm{O}$ & 1350 & 83 & L15 \\
\hline 13 & 2,5-Dimethyl-4-hydroxy-3(2H)-furanone & $3658-77-3$ & $\mathrm{C}_{6} \mathrm{H}_{8} \mathrm{O}_{3}$ & 1022 & 93 & L19 \\
\hline 14 & 5-Hexyldihydro-2(3H)-furanone & $706-14-9$ & $\mathrm{C}_{10} \mathrm{H}_{18} \mathrm{O}_{2}$ & 1383 & 85 & L10 \\
\hline 15 & 1-Hydroxy-2-butanone & $5077-67-8$ & $\mathrm{C}_{4} \mathrm{H}_{8} \mathrm{O}_{2}$ & 798 & 85 & L9 \\
\hline 16 & 9-Hydroxy-2-nonanone & $25368-56-3$ & $\mathrm{C}_{9} \mathrm{H}_{18} \mathrm{O}_{2}$ & 1295 & 83 & $\mathrm{~L} 2$ \\
\hline 17 & 1-Indanone & 83-33-0 & $\mathrm{C}_{9} \mathrm{H}_{8} \mathrm{O}$ & 1218 & 81 & L20 \\
\hline 18 & 5-Isopropylfuran-2(3H)-one & $\begin{array}{l}1315481-67- \\
4\end{array}$ & $\mathrm{C}_{7} \mathrm{H}_{10} \mathrm{O}_{2}$ & 956 & 80 & L2 \\
\hline 19 & 4-Methyl-cyclopentadecanone & $34894-60-5$ & $\mathrm{C}_{16} \mathrm{H}_{30} \mathrm{O}$ & 2031 & 85 & L6 \\
\hline 20 & 4-Methyl-2-hexanone & $105-42-0$ & $\mathrm{C}_{7} \mathrm{H}_{14} \mathrm{O}$ & 789 & 90 & L20 \\
\hline 21 & 4-Methyl-2-oxepanone & $2549-60-2$ & $\mathrm{C}_{7} \mathrm{H}_{12} \mathrm{O}_{2}$ & 1126 & 89 & L7 \\
\hline 22 & 4-Methyl-4-penten-2-one & $3744-02-3$ & $\mathrm{C}_{6} \mathrm{H}_{10} \mathrm{O}$ & 721 & 86 & L19 \\
\hline 23 & (E)-3-Octen-2-one & $18402-82-9$ & $\mathrm{C}_{8} \mathrm{H}_{14} \mathrm{O}$ & 960 & 95 & L17 \\
\hline 24 & 3-Pentylcyclopentanone & $85163-13-9$ & $\mathrm{C}_{10} \mathrm{H}_{18} \mathrm{O}$ & 1145 & 88 & L13 \\
\hline 25 & Solavetivone & $54878-25-0$ & $\mathrm{C}_{15} \mathrm{H}_{22} \mathrm{O}$ & 1645 & 85 & L11 \\
\hline 26 & Tetrahydro-6-pentenyl-pyran-2-one & 25524-95-2 & $\mathrm{C}_{10} \mathrm{H}_{16} \mathrm{O}_{2}$ & 1205 & 82 & L5 \\
\hline 27 & 2-Tridecanone & $593-08-8$ & $\mathrm{C}_{13} \mathrm{H}_{26} \mathrm{O}$ & 1449 & 94 & L15 \\
\hline 28 & $\begin{array}{l}\text { 3,3,6-Trimethyl-1,5-heptadien-4-one } \\
\text { Alcohols }\end{array}$ & $546-49-6$ & $\mathrm{C}_{10} \mathrm{H}_{16} \mathrm{O}$ & 1042 & 84 & L4 \\
\hline 29 & {$\left[\mathrm{~S}-\left(\mathrm{R}^{*}, \mathrm{R}^{*}\right)\right]-2,3-$ Butanediol } & $5341-95-7$ & $\mathrm{C}_{4} \mathrm{H}_{10} \mathrm{O}_{2}$ & 743 & 96 & L14 \\
\hline 30 & Diglycerol & 59113-36-9 & $\mathrm{C}_{6} \mathrm{H}_{14} \mathrm{O}_{5}$ & 1504 & 93 & L16 \\
\hline 31 & Glycerine & $56-81-5$ & $\mathrm{C}_{3} \mathrm{H}_{8} \mathrm{O}_{3}$ & 967 & 96 & L16 \\
\hline 32 & 1,5-Heptadiene-3,4-diol & $51945-98-3$ & $\mathrm{C}_{7} \mathrm{H}_{12} \mathrm{O}_{2}$ & 1040 & 91 & L13 \\
\hline 33 & (Z)-9-Hexadecen-1-ol & $10378-01-5$ & $\mathrm{C}_{16} \mathrm{H}_{32} \mathrm{O}$ & 1862 & 93 & L10 \\
\hline 34 & 6-Methyl-5-hepten-2-ol & $1569-60-4$ & $\mathrm{C}_{8} \mathrm{H}_{16} \mathrm{O}$ & 924 & 88 & L5 \\
\hline 35 & 6-Methyl-2-hepten-4-ol & $153665-39-5$ & $\mathrm{C}_{8} \mathrm{H}_{16} \mathrm{O}$ & 923 & 88 & L5 \\
\hline 36 & 2-Methyl-2-nonen-1-ol & 43161-19-9 & $\mathrm{C}_{10} \mathrm{H}_{20} \mathrm{O}$ & 1243 & 89 & $\mathrm{~L} 4$ \\
\hline 37 & 2-Octanol & $123-96-6$ & $\mathrm{C}_{8} \mathrm{H}_{18} \mathrm{O}$ & 1060 & 97 & L9 \\
\hline 38 & E-2-Tetradecen-1-ol & $75039-86-0$ & $\mathrm{C}_{14} \mathrm{H}_{28} \mathrm{O}$ & 1664 & 91 & L8 \\
\hline 39 & $\begin{array}{l}\text { 2,4-Undecadien-1-ol } \\
\text { Acids }\end{array}$ & $59376-58-8$ & $\mathrm{C}_{11} \mathrm{H}_{20} \mathrm{O}$ & 1373 & 92 & L15 \\
\hline 40 & 2-Decenoic acid & $3913-85-7$ & $\mathrm{C}_{10} \mathrm{H}_{18} \mathrm{O}_{2}$ & 1380 & 98 & L4 \\
\hline 41 & Dodecanoic acid & $143-07-7$ & $\mathrm{C}_{12} \mathrm{H}_{24} \mathrm{O}_{2}$ & 1570 & 97 & L8 \\
\hline 42 & Heptanoic acid & $111-14-8$ & $\mathrm{C}_{7} \mathrm{H}_{14} \mathrm{O}_{2}$ & 1074 & 97 & L13 \\
\hline 43 & 2-Heptenoic acid & $18999-28-5$ & $\mathrm{C}_{7} \mathrm{H}_{12} \mathrm{O}_{2}$ & 1081 & 96 & L4 \\
\hline 44 & (E)-3-Hexenoic acid & $1577-18-0$ & $\mathrm{C}_{6} \mathrm{H}_{10} \mathrm{O}_{2}$ & 982 & 95 & L19 \\
\hline 45 & (E)-2-Methyl-2-butenoic acid & 80-59-1 & $\mathrm{C}_{5} \mathrm{H}_{8} \mathrm{O}_{2}$ & 860 & 92 & L8 \\
\hline 46 & 2-Methyl-propanoic acid & $79-31-2$ & $\mathrm{C}_{4} \mathrm{H}_{8} \mathrm{O}_{2}$ & 711 & 89 & L8 \\
\hline 47 & (E)-2-Octenoic acid & $1871-67-6$ & $\mathrm{C}_{8} \mathrm{H}_{14} \mathrm{O}_{2}$ & 1181 & 93 & $\mathrm{~L} 4$ \\
\hline 48 & Tetradecanoic acid & $544-63-8$ & $\mathrm{C}_{14} \mathrm{H}_{28} \mathrm{O}_{2}$ & 1769 & 94 & L8 \\
\hline
\end{tabular}


Table 2. Cont.

\begin{tabular}{|c|c|c|c|c|c|c|}
\hline NO. & Volatile Compound & CAS & Formula & $\begin{array}{l}\text { Retention } \\
\text { Index }\end{array}$ & $\begin{array}{c}\text { Similarity } \\
(\%)\end{array}$ & $\begin{array}{c}\text { Recognized } \\
\text { Sample }\end{array}$ \\
\hline & Esters & & & & & \\
\hline 49 & 2-Butenoic acid, 3-methyl-, pentyl ester & $56922-72-6$ & $\mathrm{C}_{10} \mathrm{H}_{18} \mathrm{O}_{2}$ & 1168 & 84 & L15 \\
\hline 50 & Butyric acid, 1-propylpentyl ester & $20286-46-8$ & $\mathrm{C}_{12} \mathrm{H}_{24} \mathrm{O}_{2}$ & 1317 & 85 & L13 \\
\hline 51 & $\begin{array}{l}\text { Cyclobutanecarboxylic acid, } \\
\text { 2-methylpropanyl ester }\end{array}$ & $87661-19-6$ & $\mathrm{C}_{9} \mathrm{H}_{16} \mathrm{O}_{2}$ & 1141 & 82 & L15 \\
\hline 52 & Cyclobutanecarboxylic acid, 2-pentyl ester & $925444-74-2$ & $\mathrm{C}_{10} \mathrm{H}_{18} \mathrm{O}_{2}$ & 1141 & 84 & L3 \\
\hline 53 & Dibutyl phthalate & 84-74-2 & $\mathrm{C}_{16} \mathrm{H}_{22} \mathrm{O}_{4}$ & 2037 & 83 & L3 \\
\hline 54 & 1,2-Ethanediol, dipropanoate & $123-80-8$ & $\mathrm{C}_{8} \mathrm{H}_{14} \mathrm{O}_{4}$ & 1151 & 85 & L9 \\
\hline 55 & Formic acid, heptyl ester & $112-23-2$ & $\mathrm{C}_{8} \mathrm{H}_{16} \mathrm{O}_{2}$ & 1081 & 89 & L4 \\
\hline 56 & (Z)-9-Hexadecen-1-ol acetate & $34010-20-3$ & $\mathrm{C}_{18} \mathrm{H}_{34} \mathrm{O}_{2}$ & 1822 & 81 & $\mathrm{~L} 4$ \\
\hline 57 & Octanoic acid, ethyl ester & $106-32-1$ & $\mathrm{C}_{10} \mathrm{H}_{20} \mathrm{O}_{2}$ & 1183 & 89 & L17 \\
\hline 58 & Octanoic acid, pentyl ester & $638-25-5$ & $\mathrm{C}_{13} \mathrm{H}_{26} \mathrm{O}_{2}$ & 1481 & 88 & L4 \\
\hline 59 & Oxalic acid, butyl propyl ester & $26404-30-8$ & $\mathrm{C}_{9} \mathrm{H}_{16} \mathrm{O}_{4}$ & 1250 & 87 & L9 \\
\hline 60 & 2-Phenylacetic acid,2-ethylhexyl ester & $5421-30-7$ & $\mathrm{C}_{16} \mathrm{H}_{24} \mathrm{O}_{2}$ & 1758 & 88 & L4 \\
\hline 61 & $\begin{array}{l}\text { 2-Propenoic acid, tridecyl ester } \\
\text { Alkenes }\end{array}$ & 2495-25-2 & $\mathrm{C}_{17} \mathrm{H}_{32} \mathrm{O}_{2}$ & 1814 & 90 & L14 \\
\hline 62 & trans- $\alpha$-Bergamotene & $13474-59-4$ & $\mathrm{C}_{15} \mathrm{H}_{24}$ & 1430 & 81 & L11 \\
\hline 63 & 3,7-Decadiene & $72015-36-2$ & $\mathrm{C}_{10} \mathrm{H}_{18}$ & 1032 & 90 & L4 \\
\hline 64 & $\begin{array}{l}\text { Decahydro-1,1,4,7-tetramethyl-1H- } \\
\text { cycloprop[e]azulene }\end{array}$ & $6790-78-9$ & $\mathrm{C}_{15} \mathrm{H}_{26}$ & 1380 & 83 & L8 \\
\hline 65 & 3,4-Dimethylpent-1-ene & $7385-78-6$ & $\mathrm{C}_{7} \mathrm{H}_{14}$ & 1030 & 84 & $\mathrm{~L} 4$ \\
\hline 66 & $\begin{array}{l}\text { (E)-7,11-Dimethyl-3-methylene-1,6,10- } \\
\text { dodecatriene }\end{array}$ & 18794-84-8 & $\mathrm{C}_{15} \mathrm{H}_{24}$ & 1440 & 82 & L11 \\
\hline 67 & 3,3-Dimethyl-1-octene & 74511-51-6 & $\mathrm{C}_{10} \mathrm{H}_{20}$ & 921 & 88 & L1 \\
\hline 68 & 1,5-Dodecadiene & $84348-04-9$ & $\mathrm{C}_{12} \mathrm{H}_{22}$ & 1212 & 89 & L3 \\
\hline 69 & 1-Ethoxy-4,4-dimethyl-2-pentene & $55702-60-8$ & $\mathrm{C}_{9} \mathrm{H}_{18} \mathrm{O}$ & 915 & 84 & L11 \\
\hline 70 & 8-Heptadecene & $16369-12-3$ & $\mathrm{C}_{17} \mathrm{H}_{34}$ & 1719 & 89 & L10 \\
\hline 71 & 1-Heptadecyne & $26186-00-5$ & $\mathrm{C}_{17} \mathrm{H}_{32}$ & 1709 & 92 & L10 \\
\hline 72 & 10-Heneicosene & $95008-11-0$ & $\mathrm{C}_{21} \mathrm{H}_{42}$ & 2117 & 93 & L16 \\
\hline 73 & 1,15-Hexadecadiene & 21964-51-2 & $\mathrm{C}_{16} \mathrm{H}_{30}$ & 1592 & 91 & L6 \\
\hline 74 & $\begin{array}{l}\text { 1,2,3,5,6,7,8,8a-Octahydro-1,4-dimethyl-7- } \\
\text { (1-methylethenyl)-azulene }\end{array}$ & $489-81-6$ & $\mathrm{C}_{15} \mathrm{H}_{24}$ & 1490 & 81 & L11 \\
\hline 75 & $\begin{array}{c}\text { 7-Oxabicyclo[2.2.1]hept-5-ene-2,3- } \\
\text { dicarboxylic } \\
\text { anhydride }\end{array}$ & $6118-51-0$ & $\mathrm{C}_{8} \mathrm{H}_{6} \mathrm{O}_{4}$ & 1248 & 81 & L6 \\
\hline 76 & (Z)-5-Tetradecene & $41446-62-2$ & $\mathrm{C}_{14} \mathrm{H}_{28}$ & 1421 & 90 & L13 \\
\hline 77 & $\begin{array}{l}\text { 3,7,7-Trimethyl-11- } \\
\text { methylenespiro[5.5]undec-2-ene } \\
\text { Alkanes }\end{array}$ & $15401-86-2$ & $\mathrm{C}_{15} \mathrm{H}_{24}$ & 1507 & 83 & L11 \\
\hline 78 & 1-Butyl-2-ethylcyclopentane & 72993-32-9 & $\mathrm{C}_{11} \mathrm{H}_{22}$ & 999 & 84 & L15 \\
\hline 79 & 1-Cyclopropylpentane & 2511-91-3 & $\mathrm{C}_{8} \mathrm{H}_{16}$ & 819 & 82 & L13 \\
\hline 80 & $\begin{array}{l}\text { 1,1-Dimethyl-3-methylidene-2-prop-2- } \\
\text { enylidenecyclohexane }\end{array}$ & $99647-15-1$ & $\mathrm{C}_{12} \mathrm{H}_{18}$ & 788 & 83 & L2 \\
\hline 81 & 3,7-Dimethyl-nonane & $17302-32-8$ & $\mathrm{C}_{11} \mathrm{H}_{24}$ & 986 & 94 & L7 \\
\hline 82 & 1,2-Epoxydodecane & $2855-19-8$ & $\mathrm{C}_{12} \mathrm{H}_{24} \mathrm{O}$ & 1304 & 91 & L4 \\
\hline 83 & 5-Ethylundecane & 17453-94-0 & $\mathrm{C}_{13} \mathrm{H}_{28}$ & 1249 & 94 & L11 \\
\hline 84 & 3-Methyl-5-propylnonane & $31081-18-2$ & $\mathrm{C}_{13} \mathrm{H}_{28}$ & 1185 & 92 & L16 \\
\hline 85 & $\begin{array}{l}\text { (S)- }\{[4-(\text { Phenylmethoxy)phenoxy]methyl }\}- \\
\text { oxirane }\end{array}$ & $122797-04-0$ & $\mathrm{C}_{16} \mathrm{H}_{16} \mathrm{O}_{3}$ & 410 & 88 & L17 \\
\hline 86 & n-Nonylcyclohexane & $2883-02-5$ & $\mathrm{C}_{15} \mathrm{H}_{30}$ & 1576 & 89 & L10 \\
\hline 87 & $\begin{array}{l}\text { cis-2-Phenyl-1-(2-methyl-1- } \\
\text { propenyl)cyclopropane }\end{array}$ & $89486-56-6$ & $\mathrm{C}_{13} \mathrm{H}_{16}$ & 1078 & 83 & L3 \\
\hline 88 & Propyl-cyclopropane & $2415-72-7$ & $\mathrm{C}_{6} \mathrm{H}_{12}$ & 620 & 88 & $\mathrm{~L} 2$ \\
\hline 89 & 2,2,3,3-Tetramethylhexane & $13475-81-5$ & $\mathrm{C}_{10} \mathrm{H}_{22}$ & 846 & 97 & L19 \\
\hline 90 & (S)-2-Tridecyloxirane & 96938-07-7 & $\mathrm{C}_{15} \mathrm{H}_{30} \mathrm{O}$ & 1603 & 84 & L19 \\
\hline
\end{tabular}


Table 2. Cont.

\begin{tabular}{|c|c|c|c|c|c|c|}
\hline NO. & Volatile Compound & CAS & Formula & $\begin{array}{l}\text { Retention } \\
\text { Index }\end{array}$ & $\begin{array}{c}\text { Similarity } \\
(\%)\end{array}$ & $\begin{array}{c}\text { Recognized } \\
\text { Sample }\end{array}$ \\
\hline & Furans & & & & & \\
\hline 91 & Dibenzofuran & $132-64-9$ & $\mathrm{C}_{12} \mathrm{H}_{8} \mathrm{O}$ & 1483 & 90 & L20 \\
\hline 92 & Furan & $110-00-9$ & $\mathrm{C}_{4} \mathrm{H}_{4} \mathrm{O}$ & 553 & 96 & L6 \\
\hline 93 & $\begin{array}{l}\text { 2-Hexyl-2-methyl-5-(propan-2- } \\
\text { ylidene)tetrahydrofuran }\end{array}$ & 124099-79-2 & $\mathrm{C}_{14} \mathrm{H}_{26} \mathrm{O}$ & 1147 & 91 & $\mathrm{~L} 4$ \\
\hline 94 & $\begin{array}{l}\text { Octahydro-2,3'-bifuran } \\
\text { Phenols }\end{array}$ & $73373-15-6$ & $\mathrm{C}_{8} \mathrm{H}_{14} \mathrm{O}_{2}$ & 1079 & 87 & L1 \\
\hline 95 & 4-Ethyl-2-methoxy-phenol & $2785-89-9$ & $\mathrm{C}_{9} \mathrm{H}_{12} \mathrm{O}_{2}$ & 1303 & 85 & L20 \\
\hline 96 & 2-Ethylphenol & $90-00-6$ & $\mathrm{C}_{8} \mathrm{H}_{10} \mathrm{O}$ & 1114 & 89 & L20 \\
\hline 97 & 2-Methoxy-4-methyl-phenol & $93-51-6$ & $\mathrm{C}_{8} \mathrm{H}_{10} \mathrm{O}_{2}$ & 1203 & 89 & L20 \\
\hline 98 & 2,4-bis(1,1-dimethylethyl)-Phenol & $96-76-4$ & $\mathrm{C}_{14} \mathrm{H}_{22} \mathrm{O}$ & 1555 & 86 & L8 \\
\hline
\end{tabular}

L4 had the largest number of unique volatile compounds, indicating that its characteristic flavor was probably different from other samples. The unique volatile compounds of L4 were mostly concentrated in acids and esters that shows the specific flavor of flowers, iris, and fruit (octanoic acid pentyl ester). Moreover, the characteristic flavor of the L8 sample was probably weak floral, woody, and slight scented with laurel oil (dodecanoic acid). The characteristic flavor of the L20 sample might be herbs (4-ethyl-2-methoxy-phenol), spicy (2-methoxy-4-methyl-phenol), and phenol odor (2-ethyl-phenol). Meanwhile, no specific compounds were detected in L12 and L18, demonstrating that the flavor of these two samples may be more popular and have few characteristics.

Therefore, the common and unique volatile compounds reflect the basic and characteristic flavor of $\mathrm{VCO}$, respectively, and reveal the commonness and specificity of VCO flavor in different provenance areas, which is conducive to the establishment of VCO brands with local characteristics.

\subsection{Multivariate Statistical Analysis}

\subsubsection{Principal Component Analysis}

To fully understand the differences of VCO among 20 important planting areas of Camellia oleifera, a multivariate statistical analysis is applied to reveal the distribution and relationship of their volatile components. From the score plots of the principal component analysis (PCA) for ketones, alcohols, alkanes, esters, aldehydes, alkenes, acids, phenols, furans, and benzenes as shown in Figure 3A, all samples were scattered in four parts. The distribution of variability was mainly driven by the first two principal components that accounted for $80.6 \%$ of the total variance (factor 1, 60.5\%; factor 2, 20.1\%). The samples that have more specific volatile substances, such as L4, L8, and L20, were farther away from the PCA center point. Therefore, it could be inferred that the 23 common (Table 1) and 98 unique (Table 2) volatile compounds determine the particularity of VCO flavor in various planting regions.

In addition, the samples in the same group are indistinguishable. The location of variables in the loading plot explains the reasons why certain observations form clusters in the score plot [26]. By the PCA of the volatile components of VCO, the L8 and L20 oils located in the left part, while the other samples were distributed around the center. It indicates that the overall volatile profile of two oils is similar but different from the other 18 location samples. In previous research on olive oil, growing locations, such as climatic [27] and pedoclimatic conditions [25], were found to affect the odorant profiles. We believe that this conclusion is also applicable to VCO study. 
A)

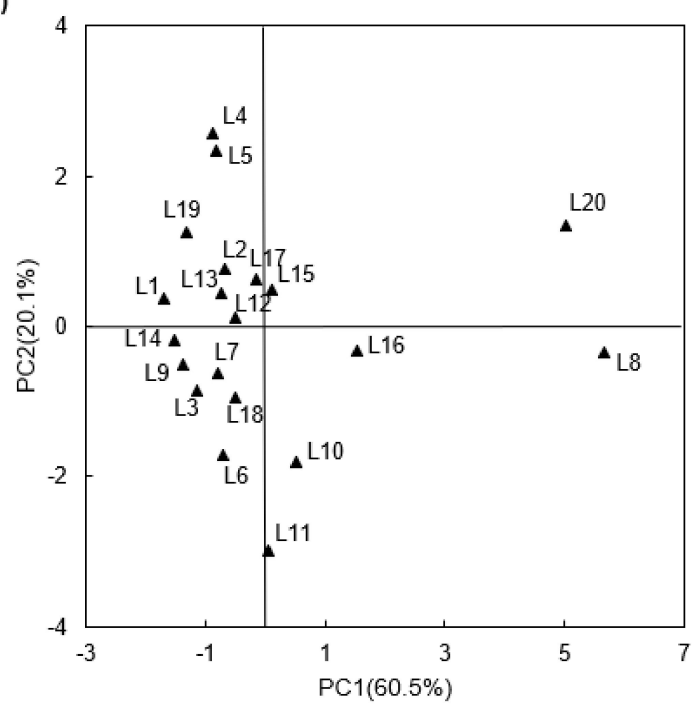

B)

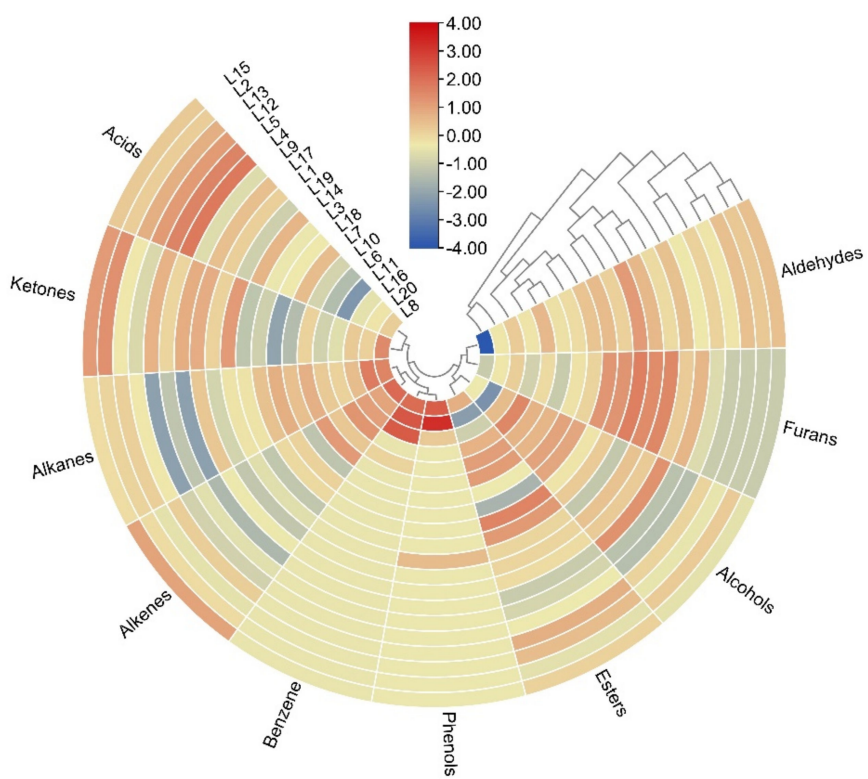

Figure 3. Principal component analysis and heatmap analysis of volatile compounds of VCO from 20 geographic regions. (A) Score plot of principal component analysis of all volatile compounds data of Camellia oleifera seed oil. (B) Heat map analysis of Camellia oil using the main types of $\mathrm{f}$ volatile composition data.

\subsubsection{Heatmap Analysis}

Heatmap is another statistical method widely used in recent years. It can aggregate many data to show the results as gradual color bands to illustrate the density and frequency of the data [1]. To investigate the variable distribution among the groups based on the PCA results, heatmap analysis of the volatile components of the 20 tested oils was employed. The results are presented as a visual heat map added to the dendrogram in Figure 3B.

According to the relative contents of volatile components, the VCO samples from 20 regions were divided into two main categories, which is consistent with the results of PCA analysis. The first included the samples of L8, L16, and L20 in which the contents of phenols, alkanes, benzene, alkenes, and ketones are relatively high. In the second main category, it could be divided into two subordinate classifications. The first included the samples of L2, L4, L5, L12, L13, and L15 that the contents of ketones, acids, and esters are comparatively high. Moreover, there were two sorts in the second subordinate classification 
that the first included L1, L3, L14, L17, and L19 oils and the contents of esters, furans, and aldehydes are high. For the rest of the oils, the contents of esters, alcohols, alkenes, and ketones are high.

\subsubsection{Partial Least Squares-Discrimination Analysis}

Partial least squares-discriminant analysis (PLS-DA) is a kind of clustering or separation method with two data matrices of $\mathrm{X}$ (explanatory dataset) and $\mathrm{Y}$ (explicative dataset) [44], which has been widely applied for biomarker selection in metabolomics [45]. Based on the PCA and heatmap analysis, PLS-DA was carried out to further classify $20 \mathrm{VCO}$ with flavor characteristics. As shown in Figure 4A, a significant discrimination according to the data matrix of their volatile compounds of VCO can be observed by using a PLSDA model. The cross-validated predictive capability $\left(\mathrm{Q}^{2}=0.268, p<0.005\right)$ indicates the model's good feasibility. The four groups were then clustered with A group for the sample dots of pink (L8, L16, and L20), B group for red (L2, L4, L5, L12, L13, and L15), C group for green (L1, L3, L14, L17, and L19), and another D group for the sample dots of purple (L6, L7, L9, L10, L11, and L18). The A group was in the lower part, whereas the B, C, and D groups accumulated in the upper part of the picture, four of which had partial overlapping. Thus, the groupings in the scatter plot are with respect to geography.

A)

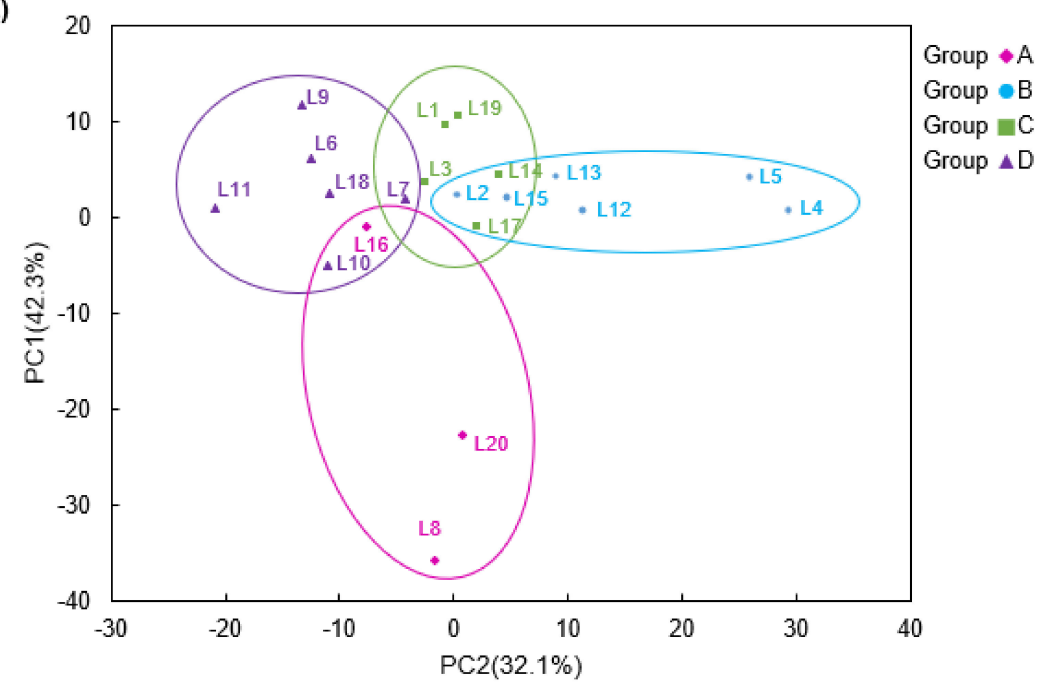

B)

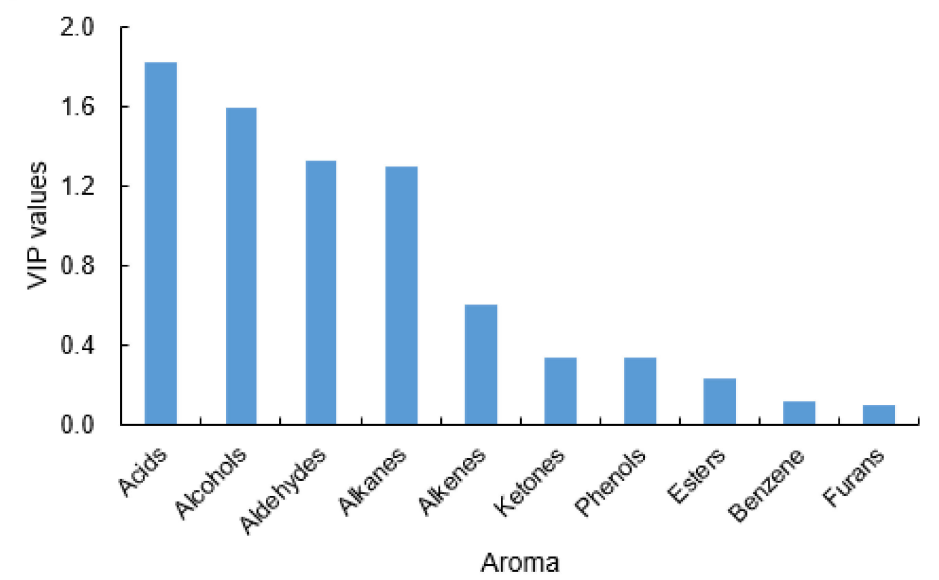

Figure 4. Partial least squares-discrimination analysis of volatile compounds of VCO from 20 geographic regions. (A) Score plot of partial least squares-discrimination analysis of all volatile compounds data of Camellia oleifera seed oil. (B) The variables important in the projection scores of 10 chemical categories of volatile compounds. 
To better understand the metabolites that affect the contribution in classification of the four groups in the PLS-DA model, values of variable importance in projection (VIP) are commonly used to calculate and identify for volatile compounds, especially in study of geographical discrimination [23]. Usually, the average VIP on a particular model is 1. When VIP exceeds 1 , the variable is considered to have important function on the PLS-DA discriminant process $[44,46]$. In Figure 4B, the variables of the PLS-DA model of volatile data with VIP values greater than 1.0 included acids, alcohols, aldehydes, and alkanes in descending order. It indicates that these compounds are potential markers for the clustering and classification of the four groups in PLS-DA score plot.

\section{Material and Methods}

\subsection{Materials}

Camellia oleifera fruits or seeds were collected from 20 geographical location in southern China, including Guangdong, Hunan, Jiangxi, Hainan Province, and Guangxi Zhuang Autonomous Region. In each area, 3 or 4 C. oleifera samples were collected. All C. oleifera fruits were harvested at almost the same maturity stage during the crop season 2020. The sampling sites were primarily selected based on the geographical location, cultivars, altitude, annual average temperature, annual rainfall, annual sunshine, and major climate types of the region, which may influence the fruit ripening capacity and quality potential (Table 3).

\subsection{Oil Extraction}

After sun exposure and manual shelling, only the $C$. oleifera seeds with no infection or those that were physically damaged were obtained from fruits and put into the oven at $75{ }^{\circ} \mathrm{C}$ for hot air drying until constant weight $[47,48]$. Then, the seeds were crushed and transferred to a BOZY-01G screw press from Hanhuang Electric Appliance Technology Co., Ltd. (Zhejiang, China). After the screw-pressing process, the crude oil was centrifuged at $10,000 \mathrm{rpm}$ for $10 \mathrm{~min}$ at $4{ }^{\circ} \mathrm{C}$. Finally, the supernatant oil as virgin C. oleifera seed oil (VCO) was kept in brown glass bottles in a cool place until analysis.

\subsection{Volatile Compounds Analysis}

\subsubsection{HS-SPME}

The volatile compounds analysis of VCO was based on the previous flavor research [18] at which point we further optimized them [49]. The $20 \mathrm{~mL}$ samples of VCO were put into $40 \mathrm{~mL}$ headspace vials, which were hermetically sealed with silicone pad. The headspace vials with oil were allowed to equilibrate for $10 \mathrm{~min}$ at room temperature. The target volatile organic substances of the samples were extracted for $33 \mathrm{~min}$ at $40^{\circ} \mathrm{C}$ using a headspace solid phase microextraction manual sampler injection handle (Shanghai Anpu Experimental Technology Co., Ltd., China) with 50/30 $\mu \mathrm{m}$ Divinylbenzene/Carboxen/Polydimethylsiloxane (DVB/CAR/PDMS) solid-phase microextraction (SPME) fiber. The volatile compounds of oils were desorbed by directly inserting SPME fiber for $3 \mathrm{~min}$ into the injection port of gas chromatography maintained at $250{ }^{\circ} \mathrm{C}$.

\subsubsection{GC-MS Analysis}

The Shimadzu QP2020 Gas Chromatography-Mass Spectrometer system (GC-MS) equipped with a flame ionization detector (FID) was used to analyze volatile components. The MS signal for the identification was simultaneously obtained by the GC system and the odor characteristics of each compound were detected by a sniffing port. An SH-Rxi-5Sil MS capillary column $(30 \mathrm{~m} \times 0.25 \mathrm{~mm} \times 0.25 \mu \mathrm{m})$ was used for analysis in GC system. The carrier gas was helium with a purity of $99.999 \%$. 
Table 3. Geographical ecological factors of different sampling sites.

\begin{tabular}{|c|c|c|c|c|c|c|c|c|c|}
\hline Samples & Collected Location & Cultivars & Latitude & Longitude & Altitude (m) & $\begin{array}{l}\text { Annual Average } \\
\text { Temperature }\left({ }^{\circ} \mathrm{C}\right)\end{array}$ & $\begin{array}{c}\text { Annual } \\
\text { Rainfall (mm) }\end{array}$ & $\begin{array}{c}\text { Annual } \\
\text { Sunshine } \\
\text { Duration (h) }\end{array}$ & Climate \\
\hline L1 & $\begin{array}{l}\text { Sihui, Zhaoqing, } \\
\text { Guangdong }\end{array}$ & $\begin{array}{c}\text { Camellia } \\
\text { semiserrata Chi }\end{array}$ & $23^{\circ} 35^{\prime} \mathrm{N}$ & $112^{\circ} 33^{\prime} \mathrm{E}$ & $\leq 1000$ & $20-22$ & 1750 & 1600 & Subtropical climate \\
\hline L2 & $\begin{array}{l}\text { Lianzhou, Qingyuan, } \\
\text { Guangdong }\end{array}$ & $\begin{array}{c}\text { Camellia } \\
\text { meiocarpa } \mathrm{Hu}\end{array}$ & $25^{\circ} 05^{\prime} \mathrm{N}$ & $112^{\circ} 37^{\prime} \mathrm{E}$ & $\leq 1000$ & $19-21$ & 1625 & 1510 & $\begin{array}{c}\text { Central Asia monsoon } \\
\text { climate }\end{array}$ \\
\hline L3 & $\begin{array}{l}\text { Qingxin, Qingyuan, } \\
\text { Guangdong }\end{array}$ & $\begin{array}{c}\text { Camellia oleifera } \\
\text { Abel }\end{array}$ & $23^{\circ} 44^{\prime} \mathrm{N}$ & $113^{\circ} 0^{\prime} \mathrm{E}$ & $\leq 1000$ & $19-21$ & 1625 & 1510 & $\begin{array}{c}\text { Central Asia monsoon } \\
\text { climate }\end{array}$ \\
\hline $\mathrm{L} 4$ & $\begin{array}{c}\text { Yangchun, Yangjiang, } \\
\text { Guangdong }\end{array}$ & $\begin{array}{l}\text { Camellia oleifera } \\
\text { Abel }\end{array}$ & $22^{\circ} 19^{\prime} \mathrm{N}$ & $111^{\circ} 51^{\prime} \mathrm{E}$ & $\leq 200$ & $21-28$ & 2380 & 2000 & $\begin{array}{l}\text { Subtropical rainforest } \\
\text { climate }\end{array}$ \\
\hline L5 & $\begin{array}{l}\text { Qujiang, Shaoguan, } \\
\text { Guangdong }\end{array}$ & $\begin{array}{c}\text { Camellia oleifera } \\
\text { Abel }\end{array}$ & $24^{\circ} 42^{\prime} \mathrm{N}$ & $113^{\circ} 49^{\prime} \mathrm{E}$ & $\leq 200$ & $18-26$ & 1700 & 1660 & $\begin{array}{l}\text { Subtropical monsoon } \\
\text { climate }\end{array}$ \\
\hline L6 & $\begin{array}{l}\text { Guangning, } \\
\text { Zhaoqing, } \\
\text { Guangdong }\end{array}$ & $\begin{array}{c}\text { Camellia oleifera } \\
\text { Abel }\end{array}$ & $23^{\circ} 39^{\prime} \mathrm{N}$ & $112^{\circ} 21^{\prime} \mathrm{E}$ & $\leq 300$ & $20-22$ & 1720 & 1613 & $\begin{array}{c}\text { Transitional climate } \\
\text { between South Asia and } \\
\text { central subtropics }\end{array}$ \\
\hline L7 & $\begin{array}{l}\text { Yunan, Yunfu, } \\
\text { Guangdong }\end{array}$ & $\begin{array}{c}\text { Camellia oleifera } \\
\text { Abel }\end{array}$ & $22^{\circ} 56^{\prime} \mathrm{N}$ & $111^{\circ} 53^{\prime} \mathrm{E}$ & $\leq 1000$ & $20-25$ & 1580 & 1480 & $\begin{array}{l}\text { Subtropical monsoon } \\
\text { climate }\end{array}$ \\
\hline L8 & $\begin{array}{l}\text { Longchuan, Heyuan, } \\
\text { Guangdong }\end{array}$ & $\begin{array}{c}\text { Camellia oleifera } \\
\text { Abel }\end{array}$ & $24^{\circ} 19^{\prime} \mathrm{N}$ & $115^{\circ} 15^{\prime} \mathrm{E}$ & $\leq 500$ & $18-27$ & 1500 & 1700 & $\begin{array}{l}\text { Subtropical monsoon } \\
\text { climate }\end{array}$ \\
\hline L9 & $\begin{array}{l}\text { Xingning, Meizhou, } \\
\text { Guangdong }\end{array}$ & $\begin{array}{c}\text { Camellia } \\
\text { meiocarpa } \mathrm{Hu}\end{array}$ & $24^{\circ} 25^{\prime} \mathrm{N}$ & $115^{\circ} 37^{\prime} \mathrm{E}$ & $\leq 400$ & $19-26$ & 1520 & 1900 & $\begin{array}{l}\text { Transitional climate } \\
\text { between South Asia and } \\
\text { central subtropics }\end{array}$ \\
\hline L10 & $\begin{array}{l}\text { Tianhe, Guangzhou, } \\
\text { Guangdong }\end{array}$ & $\begin{array}{c}\text { Camellia } \\
\text { gauchowensis } \\
\text { Change }\end{array}$ & $23^{\circ} 11^{\prime} \mathrm{N}$ & $113^{\circ} 22^{\prime} \mathrm{E}$ & $\leq 100$ & $20-28$ & 2000 & 1620 & $\begin{array}{l}\text { Subtropical marine } \\
\text { monsoon climate }\end{array}$ \\
\hline L11 & $\begin{array}{l}\text { Xuwen, Zhanjiang, } \\
\text { Guangdong }\end{array}$ & $\begin{array}{c}\text { Camellia } \\
\text { gauchowensis } \\
\text { Change }\end{array}$ & $20^{\circ} 19^{\prime} \mathrm{N}$ & $110^{\circ} 19^{\prime} \mathrm{E}$ & $\leq 100$ & $20-25$ & 2000 & 2100 & Tropical monsoon climate \\
\hline
\end{tabular}


Table 3. Cont.

\begin{tabular}{|c|c|c|c|c|c|c|c|c|c|}
\hline Samples & Collected Location & Cultivars & Latitude & Longitude & Altitude (m) & $\begin{array}{l}\text { Annual Average } \\
\text { Temperature }\left({ }^{\circ} \mathrm{C}\right)\end{array}$ & $\begin{array}{c}\text { Annual } \\
\text { Rainfall (mm) }\end{array}$ & $\begin{array}{c}\text { Annual } \\
\text { Sunshine } \\
\text { Duration (h) }\end{array}$ & Climate \\
\hline L12 & $\begin{array}{l}\text { Gaozhou, Maoming, } \\
\text { Guangdong }\end{array}$ & $\begin{array}{c}\text { Camellia } \\
\text { gauchowensis } \\
\text { Change }\end{array}$ & $21^{\circ} 42^{\prime} \mathrm{N}$ & $110^{\circ} 36^{\prime} \mathrm{E}$ & $\leq 1600$ & $20-25$ & 1900 & 1950 & $\begin{array}{l}\text { Subtropical monsoon } \\
\text { climate }\end{array}$ \\
\hline L13 & $\begin{array}{l}\text { You, Zhuzhou, } \\
\text { Hunan }\end{array}$ & $\begin{array}{c}\text { Camellia oleifera } \\
\text { Abel }\end{array}$ & $26^{\circ} 46^{\prime} \mathrm{N}$ & $113^{\circ} 09^{\prime} \mathrm{E}$ & $\leq 1400$ & $16-18$ & 1400 & NF & $\begin{array}{l}\text { Mid-subtropical humid } \\
\text { monsoon climate }\end{array}$ \\
\hline L14 & $\begin{array}{c}\text { Yuanzhou, Yichun, } \\
\text { Jiangxi }\end{array}$ & $\begin{array}{c}\text { Camellia oleifera } \\
\text { Abel }\end{array}$ & $27^{\circ} 33^{\prime} \mathrm{N}$ & $113^{\circ} 54^{\prime} \mathrm{E}$ & $\leq 1800$ & $15-20$ & 1680 & 1740 & $\begin{array}{l}\text { Mid-subtropical monsoon } \\
\text { climate }\end{array}$ \\
\hline L15 & $\begin{array}{c}\text { Zhanggong, Ganzhou, } \\
\text { Jiangxi }\end{array}$ & $\begin{array}{c}\text { Camellia oleifera } \\
\text { Abel }\end{array}$ & $24^{\circ} 29^{\prime} \mathrm{N}$ & $113^{\circ} 54^{\prime} \mathrm{E}$ & $300-500$ & $18-22$ & 1320 & NF & $\begin{array}{l}\text { Subtropical monsoon } \\
\text { climate }\end{array}$ \\
\hline L16 & $\begin{array}{c}\text { Qingshanhu, } \\
\text { Nanchang, Jiangxi }\end{array}$ & $\begin{array}{c}\text { Camellia oleifera } \\
\text { Abel }\end{array}$ & $28^{\circ} 10^{\prime} \mathrm{N}$ & $115^{\circ} 27^{\prime} \mathrm{E}$ & $\leq 1000$ & $17-18$ & 1650 & 1800 & $\begin{array}{l}\text { Subtropical monsoon } \\
\text { climate }\end{array}$ \\
\hline L17 & $\begin{array}{c}\text { Xixiangtang, } \\
\text { Nanning, Guangxi }\end{array}$ & $\begin{array}{c}\text { Camellia } \\
\text { gauchowensis } \\
\text { Change }\end{array}$ & $22^{\circ} 48^{\prime} \mathrm{N}$ & $108^{\circ} 22^{\prime} \mathrm{E}$ & $300-600$ & $20-23$ & 1300 & NF & $\begin{array}{l}\text { Subtropical monsoon } \\
\text { climate }\end{array}$ \\
\hline L19 & $\begin{array}{l}\text { Beireng, Qionghai, } \\
\text { Hainan }\end{array}$ & $\begin{array}{c}\text { Camellia } \\
\text { vietnamensis } \\
\text { Huang ex } \mathrm{Hu}\end{array}$ & $18^{\circ} 58^{\prime} \mathrm{N}$ & $110^{\circ} 7^{\prime} \mathrm{E}$ & $\leq 100$ & $27-28$ & 2040 & 2155 & Tropical monsoon climate \\
\hline $\mathrm{L} 20$ & $\begin{array}{c}\text { Panlong, Kunming, } \\
\text { Yunnan }\end{array}$ & $\begin{array}{c}\text { Camellia oleifera } \\
\text { Abel }\end{array}$ & $25^{\circ} 02^{\prime} \mathrm{N}$ & $102^{\circ} 42^{\prime} \mathrm{E}$ & $1500-2800$ & $13-18$ & 1035 & 2200 & $\begin{array}{l}\text { Subtropical highland } \\
\text { monsoon climate }\end{array}$ \\
\hline
\end{tabular}


Operation conditions were as follows: $40^{\circ} \mathrm{C}$ of the oven temperature of SH-Rxi-5Sil MS capillary column, $250{ }^{\circ} \mathrm{C}$ of the temperature of injection block, splitless of injection mode, 50 of split ratio, $1 \mathrm{~min}$ of injection time, linear speed of flow control mode, $36.1 \mathrm{~cm} / \mathrm{s}$ of line speed, $49.7 \mathrm{kpa}$ of pressure, $54.1 \mathrm{~mL} / \mathrm{min}$ of total flow rate, $1 \mathrm{~mL} / \mathrm{min}$ of column flow rate, and $3 \mathrm{~mL} / \mathrm{min}$ of purge flow rate.

The temperature first increased to $40{ }^{\circ} \mathrm{C}$, remaining for $1.5 \mathrm{~min}$, and then to $230{ }^{\circ} \mathrm{C}$ at $4{ }^{\circ} \mathrm{C} / \mathrm{min}$ with a final hold at $230^{\circ} \mathrm{C}$ for $3 \mathrm{~min}$. The GC-MS interface and ionization source temperatures were set at 280 and $230{ }^{\circ} \mathrm{C}$, respectively. The solvent delay time was $1 \mathrm{~min}$. The total program analysis time was $52 \mathrm{~min}$.

\subsubsection{Qualitative Analysis}

The qualitative analysis of the volatile compounds was processed by GC-MS software analysis to identify unknowns with the ability of peak picking, peak deconvolution, and mass spectra comparison. Automatic integration was used by peak area with 200 of peak number and $2 \mathrm{~s}$ of half peak width. The volatile compounds were identified by comparing the mass spectra with the mass spectrometry libraries of the National Institute of Standards and Technology (NIST17). The similarities of the components were all above $80 \%$. The relative percentage content of each volatile compound in VCO was calculated by area normalization method according to the peak area.

\subsection{Statistical Analysis}

All experiments were performed in triplicate. Data and charts were processed using Microsoft Office 2010. Before the multivariate chemometric methods were applied to the original data, the data were standardized by SPSS 19.0 (IBM, SPSS version 19 IBM Corp., Armonk, NY, USA). Principal component analysis (PCA) was employed to identify the main factors controlling the composition. Heatmap analysis was performed by TB Tool software (version v1.099) to analyze the relationship with the volatile compounds and the different geographical area. Partial least squares-discrimination analysis (PLS-DA) was applied to classify all of the samples according to their volatiles using MetaboAnalyst 3.5.

\section{Conclusions}

Different from other popular vegetable oils, most virgin Camellia oleifera seed oils (VCO) from distinct producing areas have unique flavor. Therefore, this study is a first in exploring the effect of volatile compounds on the geographical discrimination of VCO from the perspective of flavor. Ten chemical categories of volatile compounds, including aldehydes, ketones, alcohols, acids, esters, alkenes, alkanes, furans, phenols, and benzenes, were detected, both number and contents of which in each sample were distinguished. A total of 23 common and 98 unique volatile compounds were determined that cause the basic and characteristic flavor of VCO from different geographic origins, respectively. The 20 main producing regions of $C$. oleifera in southern China were classified into four groups according to the VCO flavor. The regions of Longchuan in Guangdong (L8), Qingshanhu in Jiangxi (L16), Panlong in Yunnan (L20) where plants of the cultivars of C. oleifera Abel. belonged to the same category, scatter plots of which were in the lower part in partial least squares-discrimination analysis compared to other groups. From the perspective of growing environments, the annual average temperatures in these three locations are relatively low (lowest at $13^{\circ} \mathrm{C}$ ) and the annual rainfalls are also low (1035-1650 mm). Moreover, another group contained six planting areas of Guangning in Guangdong (L6), Yunan in Guangdong (L7), Xingning in Guangdong (L9), Tianhe in Guangdong (L10), Xuwen in Guangdong (L11), and Xiuying in Hainan (L18) where the annual average temperatures are relatively high (highest at $29^{\circ} \mathrm{C}$ ) but the altitudes are low $(\leq 1000 \mathrm{~m})$. The influence of geography and climate on VCO flavor is highly complicated. The geographical characterization of the other two groups were not found in our study, probably due to there being some unknown factors affecting their classification. VCO from different parts of the region have their own defining characteristics that can be used in the authentication studies 
and geographical classification of China Camellia oils further to promote the development and utilization of $\mathrm{VCO}$ as an edible oil.

Author Contributions: Conceptualization, data curation, formal analysis, methodology, validation, and writing-original draft, J.W.; formal analysis, investigation, and supervision, X.T.; writingreview and editing, Q.C.; data curation, software, and validation, M.Z.; methodology, project administration, and writing-review and editing, Y.Z.; formal analysis and resources, B.X. All authors have read and agreed to the published version of the manuscript.

Funding: This research project was financially supported by Guangdong Basic and Applied Basic Research Foundation (2019A1515011904), the Forestry Science and Technology Innovational Project of Guangdong Province (2018KJCX036), and National Natural Science Foundation of China (No. 31901271).

Institutional Review Board Statement: Not applicable.

Informed Consent Statement: Not applicable.

Data Availability Statement: Data is contained within the article or supplementary material.

Conflicts of Interest: The authors declare no conflict of interest.

Sample Availability: Samples of Camellia oleifera seeds or the oil are available from the authors.

\section{References}

1. Wu, J.; Fan, X.; Huang, X.; Li, G.; Guan, J.; Tang, X.; Qiu, M.; Yang, S.; Lu, S. Effect of different drying treatments on the quality of camellia oleifera seed oil. S. Afr. J. Chem. Eng. 2021, 35, 8-13. [CrossRef]

2. Zeng, W.; Endo, Y. Effect of cultivars and geography in China on the lipid characteristics of Camellia oleifera seeds. J. Oleo Sci. 2019, 68, 1051-1061. [CrossRef]

3. Shen, M.; Zhao, S.; Zhang, F.; Huang, M.; Xie, J. Characterization and authentication of olive, camellia and other vegetable oils by combination of chromatographic and chemometric techniques: Role of fatty acids, tocopherols, sterols and squalene. Eur. Food Res. Technol. 2021, 247, 411-426. [CrossRef]

4. Wang, X.; Zeng, Q.; Verardo, V.; Del Mar Contreras, M. Fatty acid and sterol composition of tea seed oils: Their comparison by the "Fancy Tiles" approach. Food Chem. 2017, 233, 302-310. [CrossRef]

5. Zeng, W.; Endo, Y. Lipid characteristics of Camellia seed oil. J. Oleo Sci. 2019, 68, 649-658. [CrossRef]

6. Wang, X.; Zeng, Q.; Del Mar Contreras, M.; Wang, L. Profiling and quantification of phenolic compounds in Camellia seed oils: Natural tea polyphenols in vegetable oil. Food Res. Int. 2017, 102, 184-194. [CrossRef]

7. Yuan, J.; Wang, C.; Chen, H.; Zhou, H.; Ye, J. Prediction of fatty acid composition in Camellia oleifera oil by near infrared transmittance spectroscopy (NITS). Food Chem. 2013, 138, 1657-1662. [CrossRef]

8. Wei, W.; Cheng, H.; Cao, X.; Zhang, X.; Feng, F. Triacylglycerols of camellia oil: Composition and positional distribution of fatty acids. Eur. J. Lipid Sci. Technol. 2015, 118, 1254-1255. [CrossRef]

9. Chaikul, P.; Sripisut, T.; Chanpirom, S.; Sathirachawan, K.; Ditthawuthikul, N. Melanogenesis inhibitory and antioxidant effects of Camellia oleifera seed oil. Adv. Pharm. Bull. 2017, 7, 473-477. [CrossRef]

10. Lee, C.-P.; Yen, G.-C. Antioxidant activity and bioactive compounds of tea seed (Camellia oleifera Abel.) oil. J. Agric. Food Chem. 2006, 54, 779-784. [CrossRef]

11. Lee, W.-T.; Tung, Y.-Y.; Wu, C.C.; Tu, P.-S.; Yen, G.-C. Camellia oil (Camellia oleifera Abel.) modifies the composition of the gut microbiota and alleviates acetic acid-induced colitis in rats. J. Agric. Food Chem. 2018, 66, 7384-7392. [CrossRef]

12. Cheng, Y.-T.; Lu, C.-C.; Yen, G.-C. Beneficial effects of Camellia oil (Camellia oleifera Abel.) on hepatoprotective and gastroprotective activities. J. Nutr. Sci. Vitaminol. 2015, 61, 100-102. [CrossRef]

13. Bayrak, A.; Kiralan, M.; Kara, H.H. Determination of aroma profiles of olive oils from Turkish olive cultivars. J. Am. Oil Chem. Soc. 2013, 90, 1281-1300. [CrossRef]

14. Nollet, L.M. Physical Characterization and Nutrient Analysis-Aroma Compounds. In Handbook of food Analysis, 2nd ed.; CRC Press: Boca Raton, FL, USA, 2004; Volume 1, p. 717.

15. Kiritsakis, A.K. Flavor components of olive oil-a review. J. Am. Oil Chem. Soc. 1998, 75, 673-681. [CrossRef]

16. Ridolfi, M.; Terenziani, S.; Patumi, M.; Fontanazza, G. Characterization of the lipoxygenases in some olive cultivars and determination of their role in volatile compounds formation. J. Agric. Food Chem. 2002, 50, 835-839. [CrossRef]

17. Kalua, C.M.; Allen, M.S.; Bedgood, D.R.; Bishop, A.G.; Prenzler, P.D.; Robards, K. Olive oil volatile compounds, flavor development and quality: A critical review. Food Chem. 2007, 100, 273-286. [CrossRef]

18. Kesen, S.; Kelebek, H.; Selli, S. Characterization of the key aroma compounds in Turkish olive oils from different geographic origins by application of aroma extract dilution analysis (AEDA). Agric. Food Chem. 2014, 62, 391-401. [CrossRef] 
19. Ye, J.; Wang, W.; Ho, C.; Li, J.; Guo, X.; Zhao, M.; Jiang, Y.; Tu, P. Differentiation of two types of pu-erh teas by electronic nose and ultrasound-assisted extraction-dispersive liquid-liquid microextraction-gas chromatography-mass spectrometry. Anal. Methods 2016, 8, 593-604. [CrossRef]

20. Zhu, J.; Chen, F.; Wang, L.; Niu, Y.; Yu, D.; Shu, C.; Chen, H.; Wang, H.; Xiao, Z. Comparison of aroma-active volatiles in oolong tea infusions using GC-olfactometry, GC-FPD, and GC-MS. J. Agric. Food Chem. 2015, 63, 7499-7510. [CrossRef]

21. Zeng, L.; Zhou, Y.; Fu, X.; Mei, X.; Cheng, S.; Gui, J.; Dong, F.; Tang, J.; Ma, S.; Yang, Z. Does oolong tea (Camellia sinensis) made from a combination of leaf and stem smell more aromatic than leaf-only tea? Contribution of the stem to oolong tea aroma. Food Chem. 2017, 237, 488-498. [CrossRef]

22. Zhang, C.; Zhang, Q.; Zhong, C.; Guo, M. Analysis of volatile compounds responsible for kiwifruit aroma by desiccated headspace gas chromatography-mass spectrometry. J. Chromatogr. A 2016, 1440, 255-259. [CrossRef]

23. Reale, S.; Biancolillo, A.; Gasparrini, C.; Di Martino, L.; Di Cecco, V.; Manzi, A.; Di Santo, M.; D’Archivio, A.A. Geographical discrimination of bell pepper (Capsicum annuum) spices by (HS)-SPME/GC-MS aroma profiling and chemometrics. Molecules 2021, 26, 6177. [CrossRef]

24. Zarifikhosroshahi, M.; Murathan, Z.T.; Kafkas, E.; Okatan, V. Variation in volatile and fatty acid contents among Viburnum opulus L. fruits growing different locations. Sci. Hortic. 2020, 264, 109160. [CrossRef]

25. Issaoui, M.; Flamini, G.; Brahmi, F.; Dabbou, S.; Hassine, K.B.; Taamali, A.; Chehab, H.; Ellouz, M.; Zarrouk, M.; Hammami, M. Effect of the growing area conditions on differentiation between Chemlali and Chétoui olive oils. Food Chem. 2010, 119, 220-225. [CrossRef]

26. Alkan, D.; Tokatli, F.; Ozen, B. Phenolic characterization and geographical classification of commercial extra virgin olive oils produced in Turkey. J. Am. Oil Soc. 2012, 78, 261-268. [CrossRef]

27. Kiralan, M.; Ozkan, G.; Koyluoglu, F.; Ugurlu, H.A.; Bayrak, A.; Kiritsakis, A. Effect of cultivation area and climatic conditions on volatiles of virgin olive oil. Eur. J. Lipid Sci. Technol. 2012, 114, 552-557. [CrossRef]

28. Tabaszewska, M.; Rutkowska, J.; Skoczylas, Ł.; Słupski, J.; Antoniewska, A.; Smoleń, S.; Łukasiewicz, M.; Baranowski, D.; Duda, I.; Pietsch, J. Red arils of Taxus Baccata L.-A new source of valuable fatty acids and nutrients. Molecules 2021, 26, 723. [CrossRef]

29. Peng, L.; Men, S.; Liu, Z.; Tong, N.; Imran, M.; Shu, Q. Fatty acid composition, phytochemistry, antioxidant activity on seed coat and kernel of Paeonia ostii from main geographic production areas. Foods 2020, 9, 30. [CrossRef]

30. Wang, X.; Contreras, M.; Xu, D.; Jia, W.; Wang, L.; Yang, D. New insights into free and bound phenolic compounds as antioxidant cluster in tea seed oil: Distriction and contribution. LWT Food Sci. Technol. 2021, 136, 110315. [CrossRef]

31. Angerosa, F.; Servili, M.; Selvaggini, R.; Taticchi, A.; Esposto, S.; Montedoro, G. Volatile compounds in virgin olive oil: Occurrence and their relationship with the quality. J. Chromatogr. A 2004, 1054, 17-31. [CrossRef]

32. Reboredo-Rodríguez, P.; González-Barreiro, C.; Cancho-Grande, B.; Simal-Gándara, J. Dynamic headspace/GC-MS to control the aroma fingerprint of extra-virgin olive oil from the same and different olive varieties. Food Control 2012, 25, 684-695. [CrossRef]

33. Kesen, S.; Amanpour, A.; Selli, S. Comparative evaluation of the fatty acids and aroma compounds in selected Iranian nut oils. Eur. J. Lipid Sci. Technol. 2018, 120, 1-27. [CrossRef]

34. Cuicui, L.; Lixia, H. Review on volatile flavor components of roasted oilseeds and their products. Grain Oil Sci. Technol. 2018, 1, 151-156. [CrossRef]

35. Tomé-Rodríguez, S.; Ledesma-Escobar, C.A.; Penco-Valenzuela, J.M.; Priego-Capote, F. Influence of the fatty acid profile on the volatile components of virgin olive oil subjected to thermal stress. J. Sci. Food Agric. 2021, 101, 4829-4837. [CrossRef]

36. Gómez-Rico, A.; Salvador, M.D.; La Greca, M.; Fregapane, G. Phenolic and volatile compounds of extra virgin olive oil (Olea europaea L. cv. Cornicabra) with regards to fruit ripening and irrigation management. J. Agric. Food Chem. 2006, 54, 7130-7136. [CrossRef]

37. Dabbou, S.; Issaoui, M.; Brahmi, F.; Nakbi, A.; Chehab, H.; Mechri, B.; Hammami, M. Changes in volatile compounds during processing of Tunisian-style table olives. J. Am. Oil Chem. Soc. 2012, 89, 347-354. [CrossRef]

38. He, J.; Wu, X.; Zhou, Y.; Chen, J. Effects of different preheat treatments on volatile compounds of camellia (Camellia oleifera Abel.) seed oil and formation mechanism of key aroma compounds. J. Food Biochem. 2021, 45, e13649. [CrossRef]

39. Wei, C.Q.; Liu, W.Y.; Xi, W.P.; Cao, D.; Zhang, H.J.; Ding, M.; Chen, L.; Xu, Y.Y.; Huang, K.X. Comparison of volatile compounds of hot-pressed, cold-pressed and solvent-extracted flaxseed oils analyzed by SPME-GC/MS combined with electronic nose: Major volatiles can be used as markers to distinguish differently processed oils. Eur. J. Lipid Sci. Technol. 2015, 117, 320-330. [CrossRef]

40. Wang, Z.; Yuan, Y.; Hong, B.; Zhao, X.; Gu, Z. Characteristic volatile fingerprints of four chrysanthemum teas determined by HS-GC-IMS. Molecules 2021, 26, 7113. [CrossRef]

41. Burdock, G.A. Fenaroli's Handbook of Flavor Ingredients, 6th ed.; CRC Press Inc.: Boca Raton, FL, USA, 2016.

42. Chen, L.; Li, D.; Zhu, C.; Ma, X.; Rong, Y. Fatty acids and flavor components in the oil extracted from golden melon seeds. Eur. J. Lipid Sci. Technol. 2021, 123, 2000233. [CrossRef]

43. Bonvehí, J.S. Investigation of aromatic compounds in roasted cocoa powder. Eur. Food Res. Technol. 2005, 221, 19-29. [CrossRef]

44. Yang, Y.; Zhang, M.; Yin, H.; Deng, Y.; Jiang, Y.; Yuan, H.; Dong, C.; Li, J.; Hua, J.; Wang, J. Rapid profiling of volatile compounds in green teas using Micro-Chamber/Thermal Extractor combined with thermal desorption couple to gas chromatography-mass spectrometry followed by multivariate statistical analysis. LWT-Food Sci. Technol. 2018, 96, 42-50. [CrossRef]

45. Gao, J.; Wu, B.; Gao, L.; Liu, H.; Zhang, B.; Sun, C.; Chen, K. Glycosidically bound volatiles as affected by ripening stages of Satsuma mandarin fruit. Food Chem. 2018, 240, 1097-1105. [CrossRef] 
46. Giannetti, V.; Mariani, M.B.; Mannino, P.; Marini, F. Volatile fraction analysis by HS-SPME/GC-MS and chemometric modeling for traceability of apples cultivated in the Northeast Italy. Food Control 2017, 78, 215-221. [CrossRef]

47. Zhu, G.; Liu, H.; Xie, Y.; Liao, Q.; Lin, Y.; Liu, Y.; Liu, Y.; Xiao, H.; Gao, Z.; Hu, S. Postharvest processing and storage methods for Camellia oleifera seeds. Food Rev. Int. 2019, 36, 319-339. [CrossRef]

48. Huang, D.; Tao, Y.; Li, W.; Sherif, S.A.; Tang, X. Heat transfer characteristics and kinetics of Camellia oleifera seeds during hot-air drying. J. Thermal. Sci. Eng. Appl. 2020, 12, 031017. [CrossRef]

49. Wang, J.; Tang, X.; Zhang, Y.; Wang, M.; Xu, B.; Zhang, M. Optimization of headspace solid phase micro-extraction of volatile components from Camellia oleifera seeds oil by response surface methodology. Food Res. Dev. 2020, 41, 93-100. [CrossRef] 